Supporting Information for

\title{
Differences between Colloidal and Crystalline Evaporative Deposits
}

\author{
Samantha A. McBride, Rachael Skye, Kripa K. Varanasi
}

\author{
Contents of SI \\ Crystal Properties \\ Evaporation Rates \\ Area Localization Data \\ Pinning Force \\ Convection \\ Nucleation Barrier \\ Crystal Morphologies \\ Additional References
}

Tables

Table S1. Crystal solubilities as a function of temperature

Tables S2-S5. Drop evaporation rate

Tables S6-S9. Area localization and standard deviation

Table S6. Substrate properties and estimated pinning force

\section{Figures}

Figure S1. Energetics between a crystal and substrate can play a role in nucleation and growth of the crystal

Figure S2. SEM images of evaporative deposits from 60C 


\section{Crystal Properties}

In the main text, we discuss how the nucleation barriers and the propensity to crystallize is important for controlling the deposit morphologies. Here, we briefly list and discuss other properties of the saline solutions used.

Table S1. Crystal solubilities in water, and surface energy (when available)

\begin{tabular}{|c|c|c|c|c|c|c|c|c|}
\hline Crystal & $\begin{array}{c}\mathbf{C}_{\text {sat }} \text { at } \\
\mathbf{2 0}^{\circ} \mathbf{C}\end{array}$ & $\begin{array}{c}\mathbf{C}_{\text {sat }} \text { at } \\
\mathbf{4 0}^{\circ} \mathbf{C}\end{array}$ & $\begin{array}{c}\mathbf{C}_{\text {sat }} \text { at } \\
\mathbf{6 0}^{\circ} \mathbf{C}\end{array}$ & $\begin{array}{c}\mathbf{C}_{\text {sat }} \text { at } \\
\mathbf{8 0}^{\circ} \mathbf{C}\end{array}$ & $\gamma_{\text {crystal }}$ & $\rho_{\text {crystal }}$ & $\begin{array}{c}\text { Crystal } \\
\text { Structure }\end{array}$ & $\mathbf{H}_{\mathbf{f}}{ }^{\mathbf{0}}$ \\
\hline $\begin{array}{c}\text { Calcium } \\
\text { Sulfate }\end{array}$ & $2.4 \mathrm{~g} / \mathrm{L}$ & $2.6 \mathrm{~g} / \mathrm{L}$ & $2.5 \mathrm{~g} / \mathrm{L}$ & $2.3 \mathrm{~g} / \mathrm{L}$ & $\begin{array}{c}0.35 \text { to } \\
0.4 \mathrm{~J} / \mathrm{m}^{2}\end{array}$ & $\begin{array}{c}2.96 \\
\mathrm{~g} / \mathrm{cm}^{3}\end{array}$ & $\begin{array}{c}\text { Dihydrate: } \\
\text { orthorhombic }\end{array}$ & $\begin{array}{c}-1433 \\
\mathrm{~kJ} / \mathrm{mol}\end{array}$ \\
\hline $\begin{array}{c}\text { Calcium } \\
\text { Iodate }\end{array}$ & $2.4 \mathrm{~g} / \mathrm{L}$ & $4.8 \mathrm{~g} / \mathrm{L}$ & $6.1 \mathrm{gL}$ & $6.7 \mathrm{~g} / \mathrm{L}$ & & $\begin{array}{c}4.52 \\
\mathrm{~g} / \mathrm{cm}^{3}\end{array}$ & $\begin{array}{c}\text { Anhydrous: } \\
\text { monoclinic } \\
\text { monohydrate: } \\
\text { monoclinic }\end{array}$ & \\
\hline $\begin{array}{c}\text { Silver } \\
\text { Sulfate }\end{array}$ & $7.8 \mathrm{~g} / \mathrm{L}$ & $9.6 \mathrm{~g} / \mathrm{L}$ & $11 \mathrm{~g} / \mathrm{L}$ & 13.3 & & $\begin{array}{c}5.45 \\
\mathrm{~g} / \mathrm{cm}^{3}\end{array}$ & $\begin{array}{c}\text { Dihydrate: } \\
\text { Orthorhombic }\end{array}$ & $\begin{array}{c}-716 \\
\mathrm{~kJ} / \mathrm{mol}\end{array}$ \\
\hline
\end{tabular}

Crystallization is a complex process, in which multiple polymorphs may emerge depending the crystallization conditions. While we show in the main text that this does not necessarily contribute to deposit morphology, we briefly review the various polymorphs of the relevant salts.

Gypsum, the dihydrate form of Calcium sulfate, is the stable polymorph at ambient conditions, and remains so until about $50^{\circ} \mathrm{C}$. Above $50^{\circ} \mathrm{C}$, anhydrous $\mathrm{CaSO}_{4}$ becomes dominant. However, although it is the dominant polymorph, it does not form spontaneously during precipitation below $120^{\circ} \mathrm{C}$ due to kinetic limitations. ${ }^{1}$ A metastable hemi-hydrate form known as bassanite also exists. The anhydrite form has a higher surface energy $\left(0.090 \mathrm{~J} / \mathrm{m}^{2}\right)$ than gypsum $\left(0.040 \mathrm{~J} / \mathrm{m}^{2}\right)$ at $80^{\circ} \mathrm{C}^{2}$ There are many uncertainties in the phase diagram and kinetics of the transitions between the three calcium sulfate polymorphs; and while it may be of interest to determine whether or not crystal growth in contact with substrates of varying surface energy influence the transition temperature and kinetics, it is beyond the scope of the current study.

Lauterite, the anhydrous form of calcium iodate, is naturally occurring and often found near gypsum veins. Brueggenite is a hydrate version. Little work has explored the transition between the two. 


\section{Evaporation Rates}

Table S2. Evaporation time from start to finish in minutes for different solutions on the substrates at $20^{\circ} \mathrm{C}$

\begin{tabular}{|l|c|c|c|c|c|}
\hline $\mathbf{2 0}^{\circ} \mathbf{C}$ & Water & Particles & $\mathbf{A g S O}_{4}$ & $\mathbf{C a}\left(\mathbf{I O}_{3}\right)_{\mathbf{2}}$ & $\mathbf{C a S O}_{\mathbf{4}}$ \\
\hline TOPS & 10 & 12 & 17.5 & 15.5 & 16 \\
AMS & 23 & 20 & 29.5 & 26.5 & 27.5 \\
DTS & 30 & 31 & 41.5 & 38 & 38.5 \\
OTS & 34 & 35 & 42.5 & 40 & 38 \\
FS & 32 & 33 & 38.5 & 37 & 38.5 \\
\hline
\end{tabular}

Table S3. Evaporation time from start to finish in minutes for different solutions on the substrates at $40^{\circ} \mathrm{C}$

\begin{tabular}{|l|c|c|c|c|c|}
\hline $\mathbf{4 0}^{\circ} \mathbf{C}$ & Water & Particles & $\mathbf{A g S O}_{\mathbf{4}}$ & $\mathbf{C a}\left(\mathbf{I O}_{3}\right)_{\mathbf{2}}$ & $\mathbf{C a S O}_{4}$ \\
\hline TOPS & 3.98 & 3.92 & 3.72 & 3.67 & 3.98 \\
AMS & 5.33 & 5.17 & 5.23 & 5.03 & 5.33 \\
DTS & 9.2 & 8.78 & 8.08 & 8.97 & 8.78 \\
OTS & 10.8 & 10 & 10.2 & 9.8 & 11.8 \\
FS & 11.01 & 10.08 & 10.8 & 10 & 12.5 \\
\hline
\end{tabular}

Table S4. Evaporation time from start to finish in minutes for different solutions on the substrates at $60^{\circ} \mathrm{C}$

\begin{tabular}{|l|c|c|c|c|c|}
\hline $\mathbf{6 0}^{\circ} \mathbf{C}$ & Water & Particles & $\mathbf{A g S O}_{4}$ & $\mathbf{C a}\left(\mathbf{I O}_{3}\right)_{2}$ & $\mathbf{C a S O}_{4}$ \\
\hline TOPS & 1.08 & 1.58 & 1.65 & 1.48 & 1.39 \\
AMS & 2.30 & 2.36 & 2.20 & 2.35 & 2.24 \\
DTS & 3.87 & 4.23 & 3.67 & 3.53 & 4.03 \\
OTS & 3.83 & 4.02 & 3.67 & 3.97 & 3.47 \\
FS & 3.93 & 3.95 & 4.13 & 4.72 & 3.58 \\
\hline
\end{tabular}

Table S5. Evaporation time from start to finish in seconds for different solutions on the substrates at $80^{\circ} \mathrm{C}$

\begin{tabular}{|l|c|c|c|c|c|}
\hline $\mathbf{8 0}^{\circ} \mathbf{C}$ & Water & Particles & $\mathbf{A g S O}_{4}$ & $\mathbf{C a}\left(\mathbf{I O}_{3}\right)_{2}$ & $\mathbf{C a S O}_{4}$ \\
\hline TOPS & 0.52 & 0.61 & 0.47 & 0.58 & 0.54 \\
AMS & 1.16 & 0.95 & 0.88 & 0.92 & 1.63 \\
DTS & 1.38 & 1.48 & 1.50 & 1.55 & 1.41 \\
OTS & 1.61 & 1.43 & 1.68 & 1.63 & 1.48 \\
FS & 1.79 & 1.75 & 1.90 & 1.79 & 1.90 \\
\hline
\end{tabular}




\section{Area Localization}

Standard deviations for the data shown in Figure 4 were not included in the figure as error bars in order to not obfuscate the data on the small plots. Instead, standard deviation is reported here in Tables S6-S9.

Table S6. Area localization and standard deviation at $20^{\circ} \mathrm{C}$

\begin{tabular}{|l|c|c|c|c|c|c|c|c|}
\hline $\mathbf{2 0}^{\circ} \mathbf{C}$ & Particles & stnd. dev & AgSO $_{4}$ & stnd. dev & $\mathbf{C a}_{\left(\mathbf{I O}_{3}\right)_{2}}$ & stnd. dev & CaSO $_{4}$ & stnd. dev \\
\hline TOPS & 0.967 & & 0.948 & & 1 & & 1 & \\
AMS & 1.015 & & 1 & & 0.609 & & 0.838 & \\
DTS & 0.061 & & 0.197 & & 0.367 & & 0.280 & \\
OTS & 0.066 & & 0.036 & & 0.475 & & 0.327 & \\
FS & 0.259 & & 0.099 & & 0.583 & & 0.309 & \\
\hline
\end{tabular}

Table S7. Area localization and standard deviation at $40^{\circ} \mathrm{C}$

\begin{tabular}{|l|cc|c|c|c|c|c|c|}
\hline $\mathbf{4 0}^{\circ} \mathbf{C}$ & Particles & stnd. dev & AgSO $_{4}$ & stnd. dev & $\mathbf{C a}_{\left(\mathbf{I O}_{3}\right)_{\mathbf{2}}}$ & stnd. dev & CaSO $_{4}$ & stnd. dev \\
\hline TOPS & 0.965 & 0.05 & 0.981 & 0.023 & 1.033 & 0.099 & 0.93 & 0.050 \\
AMS & 1.002 & 0.002 & 0.995 & 0.008 & 0.974 & 0.010 & 0.98 & 0 \\
DTS & 0.084 & 0.021 & 0.191 & 0.158 & 0.301 & 0.198 & 0.23 & 0.119 \\
OTS & 0.085 & 0.046 & 0.178 & 0.109 & 0.200 & 0.050 & 0.25 & 0.056 \\
FS & 0.171 & 0.023 & 0.159 & 0.086 & 0.334 & 0.043 & 0.35 & 0.049 \\
\hline
\end{tabular}

Table S8. Area localization and standard deviation at $60^{\circ} \mathrm{C}$

\begin{tabular}{|l|c|c|c|c|c|c|c|c|}
\hline $\mathbf{6 0}^{\circ} \mathbf{C}$ & Particles & stnd. dev & AgSO $_{4}$ & stnd. dev & $\mathbf{C a}_{\left(\mathbf{I O}_{3}\right)_{2}}$ & stnd. dev & $\mathbf{C a S O}_{\mathbf{4}}$ & stnd. dev \\
\hline TOPS & 1.016 & 0 & 0.88 & 0.18 & 0.999 & 0.0067 & 1.024 & 0.011 \\
AMS & 0.974 & 0 & 0.962 & 0.0063 & 1.02 & 0.009 & 0.946 & 0.02 \\
DTS & 0.095 & 0.105 & 0.09 & 0.023 & 0.122 & 0.0276 & 0.225 & 0.0616 \\
OTS & 0.119 & 0.027 & 0.084 & 0.0068 & 0.162 & 0.0036 & 0.25 & 0.136 \\
FS & 0.211 & 0.022 & 0.141 & 0.004 & 0.247 & 0.0096 & 0.478 & 0.131 \\
\hline
\end{tabular}

Table S9. Area localization and standard deviation at $80^{\circ} \mathrm{C}$

\begin{tabular}{|l|c|c|c|c|c|c|c|c|}
\hline $\mathbf{8 0}^{\circ} \mathbf{C}$ & Particles & stnd. dev & AgSO $_{4}$ & stnd. dev & $\mathbf{C a}_{\left(\mathbf{I O}_{3}\right)_{2}}$ & stnd. dev & $\mathbf{C a S O}_{\mathbf{4}}$ & stnd. dev \\
\hline TOPS & 1.04 & 0.051 & 0.98 & 0.008 & 1.06 & 0.046 & 1.06 & 0.039 \\
AMS & 1.00 & 0.003 & 1 & 0.01 & 0.996 & 0.002 & 0.608 & 0.220 \\
DTS & 0.604 & 0.175 & 0.168 & 0.030 & 0.188 & 0.020 & 0.168 & 0.037 \\
OTS & 0.730 & 0.093 & 0.154 & 0.07 & 0.247 & 0.18 & 0.260 & 0.032 \\
FS & 0.470 & 0.025 & 0.176 & 0.056 & 0.169 & 0.025 & 0.470 & 0.025 \\
\hline
\end{tabular}




\section{Pinning Force}

Table S2. Pinning force for substrates, taken for surface tension $=70 \mathrm{mN} / \mathrm{m}$.

\begin{tabular}{|c|c|c|c|c|c|c|c|}
\hline Full name & Abbr. & $\theta_{\mathrm{A}}$ & $\theta_{\mathbf{E}}$ & $\theta_{\mathrm{R}}$ & $\Delta \theta$ & $\boldsymbol{R}_{\boldsymbol{O}}$ & $F_{p i n}(\mathrm{mN})$ \\
\hline triethoxyphenylsilane & TOPS & $40^{\circ}$ & $34^{\circ}$ & $12^{\circ}$ & $28^{\circ}$ & $34^{\circ}$ & 0.14 \\
\hline $\begin{array}{l}\text { (3-aminopropyl) } \\
\text { trimethyoxysilane }\end{array}$ & AMS & $57^{\circ}$ & $54^{\circ}$ & $31^{\circ}$ & $26^{\circ}$ & $54^{\circ}$ & 0.21 \\
\hline $\begin{array}{l}1,2- \\
\text { Dichlorotetramethylsilane }\end{array}$ & DTS & $82^{\circ}$ & $68^{\circ}$ & $62^{\circ}$ & $20^{\circ}$ & $68^{\circ}$ & 0.11 \\
\hline Octyltrichlorosilane & OTS & $112^{\circ}$ & $106^{\circ}$ & $98^{\circ}$ & $14^{\circ}$ & $106^{\circ}$ & 0.07 \\
\hline $\begin{array}{l}\text { Trichloro }(1 \mathrm{H}, 1 \mathrm{H}, 2 \mathrm{H}, 2 \mathrm{H}- \\
\text { perfluorooctyl) silane }\end{array}$ & FS & $124^{\circ}$ & $110^{\circ}$ & $100^{\circ}$ & $24^{\circ}$ & $110^{\circ}$ & 0.08 \\
\hline
\end{tabular}

Scatter in the $\mathrm{x}$-axis of Figure $5 \mathrm{~b}$ is due to different values of surface tension for different temperatures. 


\section{Convection}

The saline Rayleigh number as defined by Kang et. al is: ${ }^{3}$

$$
R a_{s}=\frac{g \beta_{s} C_{o} r_{o}^{3}}{v D_{s}}
$$

Where $\beta_{s}$ is the solutal expansion coefficient $\left(\beta_{s}=\frac{1}{\rho} \frac{\partial \rho}{\partial c} \sim \frac{1}{\rho}\right.$ if we make a reasonable assumption that the fluid density $\rho$ increases linearly with added mass due to concentration), $D_{s}$ is the diffusion of salt in water, $r_{o}$ is the relevant length scale (drop radius here), $C_{o}$ is the salt concentration, and $v$ is viscosity. Using an initial concentration of $2.4 \mathrm{~g} / \mathrm{L}$ we approximate the solutal Rayleigh number for our system as 23.5 (for $r_{o}=10^{-3} \mathrm{~m}, \mathrm{~g}=9.81 \mathrm{~m} / \mathrm{s}^{2}, C_{o}=2.4 \mathrm{~g} / \mathrm{L}, \beta_{S} \sim 1 / \rho_{\text {water }}=10^{-3} \mathrm{~g} / \mathrm{L}^{-1}, v=10^{-3} \mathrm{~m}^{2} / \mathrm{s}, D_{S}=10^{-9} \mathrm{~m}^{2} / \mathrm{s}$; temperature relatively arbitrary). The thermal Rayleigh number is:

$$
R a=\frac{g \beta \Delta T r_{0}^{3}}{v \alpha}
$$

Where $\beta$ is the thermal expansion coefficient $\left(0.0002141 /{ }^{\circ} \mathrm{C}\right)$ and $\alpha$ is the thermal diffusivity $\left(0.143^{*} 10^{-6}\right.$ $\mathrm{m}^{2} / \mathrm{s}$ at $20^{\circ} \mathrm{C}, 0.163^{*} 10^{-6} \mathrm{~m}^{2} / \mathrm{s}$ at $80^{\circ} \mathrm{C}$ ). At $40^{\circ} \mathrm{C}, R a \sim 0.28$, and at $80^{\circ} \mathrm{C}, R a \sim 0.77$. The ratio of the saline to thermal Rayleigh numbers becomes:

$$
R_{T S}=\frac{\beta_{S} C_{o} \alpha}{\beta \Delta T D_{S}}
$$

Which has a value of 30-100, indicating that saline convection due to concentration polarization may dominate over thermal convection. An interesting future problem could be to differentiate the effects of thermal convection and solutal convection using salts that are even more sparingly soluble than those used here (for example, calcium carbonate) by investigating the limit in which this ratio is approximately 1 .

Another consideration in which convection within the drop is important is for justifying the use of an average supersaturation in equation 2 in the main text. During evaporation, there is likely to be concentration polarization at the air/water interface due to accumulation of non-volatile ions at that point. However, the drop also undergoes rapid convection. Because the Rayleigh number is the ratio of free convection to momentum diffusivity (viscosity), we can estimate the extent of concentration polarization by multiplying this number times the ratio of viscosity to diffusivity. This new dimensionless number is a Peclet-like number:

$$
P e=\frac{g \beta_{s} C_{o} r_{o}^{3}}{D_{S}^{2}}
$$

Because $D_{s} \sim 10^{-9} \mathrm{~m}^{2} / \mathrm{s}$, and $v \sim 10^{-3} \mathrm{~m}^{2} / \mathrm{s}$, this number will be a factor of $10^{6}$ larger than the equation $\mathrm{S} 1$, and a factor of at least $10^{3}$ greater than equation S2. Thus, we can reasonably assume that convection, whether by thermal gradients or concentration gradients, is larger than diffusivity of ions. 


\section{Nucleation Barrier}

In Equation 1 in the main text, we present a form for the Gibbs free energy barrier for nucleation for a crystal nucleating on a surface from a solution. Nucleation occurs as a result of competing processes: first, there is a negative (i.e., favorable) energy change associated with formation of the solid volume as a result of supersaturation or undercooling. Second, there is a positive energy associated with the formation of a new interface. Adding these two energies gives the free energy change of homogeneous nucleation as: ${ }^{4,5}$

$$
\Delta G_{n, h o m}=-V_{S} \Delta G_{C L}+A_{S} \gamma_{C L}
$$

(See Mullin, equation 5.1) Where $V_{S}$ is the volume of the new solid particle, $G_{C L}$ is the free energy change for the solid crystal-liquid transformation, $A_{C}$ is the interfacial area of the solid crystal particle, and $\gamma_{C L}$ is the surface tension between the liquid solvent and newly formed crystal. Under the common assumption of a spherical nucleus this equation becomes:

$$
\Delta G_{n, h o m}=-\frac{4}{3} \pi r^{3} \Delta G_{C L}+4 \pi r^{2} \gamma_{C L}
$$

Where $r$ is the radius of a spherical nucleus. The free energy change for the solid-liquid transformation in solution is a function of the activity of the solvent:

$$
\Delta G_{C L}=\frac{k_{B} T}{V_{M}} \ln a / a_{S}
$$

Where $V_{m}$ is molecular volume ( $\left.1 / \mathrm{mol}\right) k_{B}$ is Boltzmann's constant, T is temperature in Kelvin, $a$ is activity, and $a_{s}$ is the activity at the solubility level (See Kashchiev, equation 2.11 and 2.14; or Mullin equation 5.7). For ionic solutions at low concentrations levels (which is very much the case for the present work with sparingly soluble salts), activity is well approximated as molar concentration. Because supersaturation, $S$, is equal to the ratio of the concentration over the solubility concentration, this becomes:

$$
\Delta G_{C L}=\frac{k_{B} T}{V_{m}} \ln S
$$

Under the spherical nucleus assumption, it is then straightforward to derive a critical radius at which Gibbs free energy of formation (i.e., equation S6) has a maximum:

$$
r_{\text {hom }}^{*}=\frac{2 \gamma_{C L}}{\Delta G_{C L}}=\frac{2 \gamma_{C L} V_{m}}{k_{B} T \ln S}
$$

where $r *$ is the critical nucleation radius, representing the smallest required size of a nucleus with favorable growth kinetics. Nuclei larger than this radius will continue to grow, while dissolution is more favorable for nuclei smaller than this critical value. Because of the importance of nucleus size in determining growth, achieving a sufficiently large nucleation rate is fundamental to forming crystal nuclei that won't dissolve. The Gibbs energy at the critical radius gives the maximum energy barrier to crystallization. For a spherical nucleus, this has the form: 


$$
\Delta G_{n, h o m}^{*}=\frac{16 \pi \gamma_{C L}^{3} V_{m}^{2}}{3\left(k_{B} T \ln S\right)^{2}}
$$

Next, we wish to adjust this description for heterogeneous nucleation by considering the change in interfacial energies for a crystal (C) growing on a surface (S) surrounded by liquid (L). The generic form for the change in Gibbs energy due to the changing interfacial areas is:

$$
\Delta G_{\text {surface }}=A_{C S} \gamma_{C S}+A_{C L} \gamma_{C L}-A_{C S} \gamma_{S L}
$$

If we assume that the burgeoning crystal nuclei is approximately hemispherical, then $A_{C S}=\pi r^{2}$ and $A_{C L}=\frac{1}{2} A_{\text {sphere }}=2 \pi r^{2}$. Adding the volumetric contribution $\left(-V_{S} \Delta G_{C L}\right.$, where $V_{S}=\frac{2}{3} \pi r^{3}$ for a hemisphere), we arrive at an equation for the free energy change for heterogeneous nucleation: ${ }^{5}$

$$
\Delta G_{n, h e t}=-\frac{2}{3} \pi r^{3} \Delta G_{C L}+\pi r^{2}\left(2 \gamma_{C L}+\gamma_{C S}-\gamma_{S L}\right)
$$

Using the form of $\Delta G_{C L}$ from equation S8 and performing the same analysis, we can find the critical radius size and critical free energy for heterogenous nucleation:

$$
\begin{gathered}
r_{\text {het }}^{*}=\frac{\left(2 \gamma_{C L}+\gamma_{C S}-\gamma_{S L}\right) V_{m}}{k_{B} T \ln S} \\
\Delta G_{n, \text { het }}^{*}=\frac{\pi V_{m}^{2}}{3\left(k_{B} T \ln S\right)^{2}}\left(2 \gamma_{C L}+\gamma_{C S}-\gamma_{S L}\right)^{3}
\end{gathered}
$$

Which is presented as equation 1 in the main text. For crystal nuclei that are not hemispherical but can still be treated as some variation of a "spherical cap" shape, the form of the free energy associated with surface energy change can be approximated as: ${ }^{4,6}$

$$
\Delta G_{n, \text { het }}^{*}=\frac{4 \pi V_{m}^{2}}{3\left(k_{B} T \ln S\right)^{2}} \gamma_{C L}^{3}(2+\cos \theta)(1-\cos \theta)^{2}
$$

Where $\theta$ is the "contact angle" between the growing nuclei and the surface. This angle can be described as a function of the interfacial energies by making an analogy between crystal growth on a substrate surrounding by a liquid phase to drop nucleation on a substrate surrounding by a vapor phase (see Figure $\mathrm{S} 1$ on next page). The analogous version of Young's contact angle equation for a solid substrate (S), crystal nuclei (C), and liquid phase (L) is (See Mullin, equation 5.22): ${ }^{4}$

$$
\gamma_{S L}=\gamma_{C S}+\gamma_{C L} \cos \theta
$$

Regardless of which equation is used, we can see how the nucleation barrier for a growing salt crystal on a substrate from a solution depends on both the supersaturation and on the different components of the surface energies between phases. Although equation S15 is more general, the explicit roles of each component of surface energy is more readily understood from equation S14. 


\section{Crystal Morphologies}

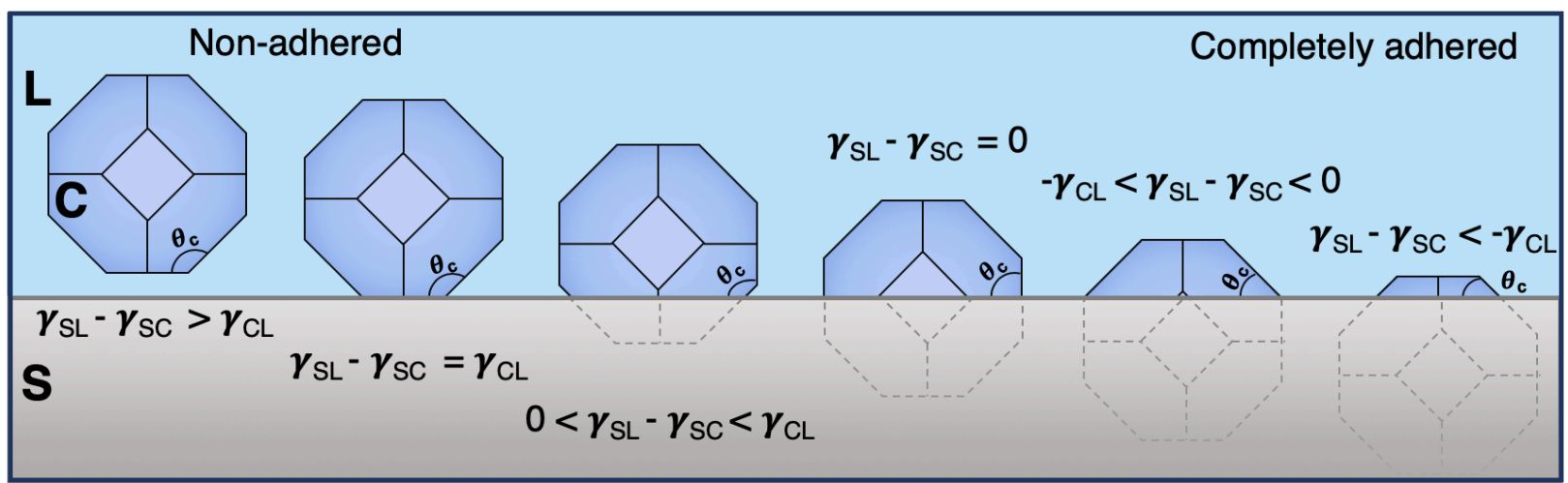

decreasing $\gamma_{\mathrm{SL}}$, increasing $\gamma_{\mathrm{SC}}$

Figure S1. The energetics between the liquid (L), substrate (S), and crystal (C) dictate the morphology of crystals and the degree of adhesion.

Equation S16 presents an analogy between the Young's contact angle equation for a drop on a surface and a growing crystal. However, the "contact angle" between a crystal and substrate is less precisely defined for a solid than it is for a liquid drop, as crystalline solids adapt preferred orientations. Predicting the equilibrium, lowest energy, nuclei shape for a crystal particle in contact with a surface as a function of the interfacial energies is known as a Winterbottom construction. ${ }^{7}$ Figure S1 shows an idealized version of how changing interfacial energies can alter the appearance and adhesion of a crystal nuclei at a surface without changing the preferred morphology of the base crystal.

In the present work, many factors influence the morphology of both the evaporative deposit and of the individual crystals that make up these deposits (See Figure S2). Both the nucleation barrier and drop wetting depend on surface energy. The nucleation barrier and wetting are controlled by volume loss, creating a complex relationship that ultimately determines the shape of the evaporative deposit. In addition to altering the preference of the system for forming a ring vs. a clumped deposit, we might also expect that surface energies can influence the morphology of the individual crystals formed. The different crystal faces within an equilibrium crystal morphology have different energetics; and therefore different crystal faces might preferentially grow on substrates of different surface energies (As shown in an idealized form in Figure S1) and form different crystal morphologies across the different substrates.

Another factor controlling crystal morphology is the supersaturation of the solution during formation, where very large values of supersaturation can fundamentally change the preferred orientation and polymorph of the crystal formed. ${ }^{8}$ As we show in Figure 5 in the main text, crystals will nucleate at different values of supersaturation depending on the substrate. Supersaturation is generally higher for highly condensed deposits formed on the most hydrophobic substrates (OTS, FS), and lower for the ringlike deposits formed on the hydrophilic substrates (TOPS, AMS). Decoupling the effects on individual crystal morphology of supersaturation from those of surface energy is exceptionally challenging due to the inter-relatedness of the two. 


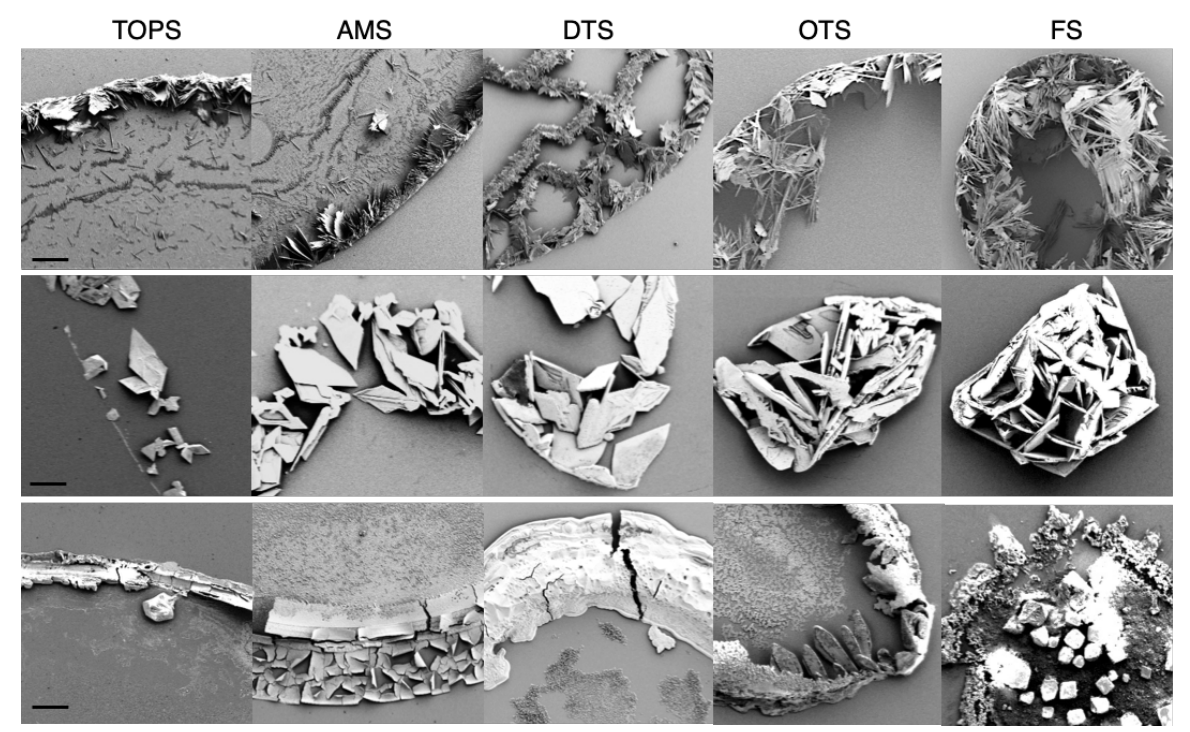

Figure S2. Influence of surface energy on deposit morphology formed at $60^{\circ} \mathrm{C}$ for (top) calcium sulfate, (middle) silver sulfate, and (bottom) calcium iodate.

In Figure S2, we see that the size of individual silver sulfate crystals increases with increasing contact angle. This observation can be rationalized by lowered evaporation times with increasing contact angles, as crystals grown at slower rates tend towards larger grain size. This effect is particularly potent for the calcium iodate crystals, in which structures formed at lower wettability's do not grow distinct facets. The kinetics of crystallization for calcium iodate are significantly slower than for the other two crystals; meaning that without sufficient time for crystal formation the calcium iodate instead precipitates in a random and condensed fashion, as shown in Figure S2.

While silver sulfate crystals have similar "arrow-like" morphologies across the different substrates, the morphologies of the calcium sulfate crystals are variable. On the TOPS, AMS, and DTS surfaces, we see more "leaf-like" morphologies, while crystals formed on the OTS and FS surfaces trend towards needle shapes. Both shapes are common morphologies for gypsum crystals, and the transition between the two with increasing contact angle is likely a combination of the previously-mentioned interfacial energy and supersaturation effects. Needle-like crystals may be more likely to form on the hydrophobic surfaces due to interfacial energies promoting a more detached crystal shape (left side of Figure S1). In addition, the supersaturation at crystallization is higher on the more hydrophobic surfaces, which can also promote a transition to needle-like gypsum morphologies. ${ }^{9}$ Decoupling the effects could be an interesting venue for future work. 


\section{Additional References}

1. Ritschler, U., Vandriessche, A. E. S., Kempter, A., Kellermeier, M. \& Cçlfen, H. Materials Chemistry Controlling the Selective Formation of Calcium Sulfate Polymorphs at Room

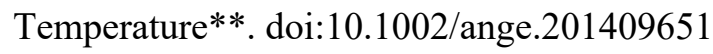

2. Freyer, D. \& Voigt, W. Crystallization and Phase Stability of CaSO 4 and CaSO 4 - Based Salts. Monatsheftef r Chemie / Chem. Mon. 134, 693-719 (2003).

3. Kang, K. H., Lim, H. C., Lee, H. W. \& Lee, S. J. Evaporation-induced saline Rayleigh convection inside a colloidal droplet. Phys. Fluids 25, 042001 (2013).

4. Mullin, J. W. Crystallization, 4th Edition. (2001).

5. De Yoreo, J. J. \& Vekilov, P. G. Principles of Crystal Nucleation and Growth. Rev. Mineral. Geochemistry 54, 57-93 (2003).

6. Volmer, M. Kinetics of Phase Formation. Die Chem. Reaktion 4, (1939).

7. Enterkin, J. A., Poeppelmeier, K. R. \& Marks, L. D. Oriented Catalytic Platinum Nanoparticles on High Surface Area Strontium Titanate Nanocuboids. Nano Lett. 11, 993-997 (2011).

8. Desarnaud, J., Derluyn, H., Carmeliet, J., Bonn, D. \& Shahidzadeh, N. Hopper Growth of Salt Crystals. J. Phys. Chem. Lett. 9, 2961-2966 (2018).

9. McBride, S. A., Dash, S. \& Varanasi, K. K. Evaporative Crystallization in Drops on Superhydrophobic and Liquid-Impregnated Surfaces. Langmuir 34, 12350-12358 (2018). 